\title{
Radio Frequency Energy Harvesting for Cell Phones
}

\author{
Hussein KASSEM \\ Assistant Professor \\ Department of EEE \\ International University of Beirut \\ (BIU), Beirut, Lebanon
}

\author{
Hayssam El-Hajj, Mohammad S \\ Abou Chahine, Ismail El-Sayad* \\ Department of EEE, *CCE \\ International University of Beirut \\ (BIU), Beirut, Lebanon
}

\author{
Ahmad Hassan. Qassab, \\ Mohammad Kassem. Salman \\ Lebanese International University \\ (LIU), Beirut, Lebanon
}

\begin{abstract}
This thesis attempts to validate that radio frequency energy harvesting for mobile phones is valid. (System design consist of 4 main parts which are proposed and implemented in their detailed information's and simulations to use the optimum and best performance parts.) First part is the antenna circuit which captures the RF signals from the mobile and wi-fi bands in atmosphere. Second part is the impedance network that matches between the RF source and the load to obtain the maximum power. After that, rectification circuit is implemented to convert RF signals to dc output voltage. At last, voltage converter is designed to step up the voltage to match the power need load. Similar work and projects are discussed to analyze the best optimum harvester that can be used. In this research, the harvester is designed and simulated using programs, which analyze the performance of the parts and the efficient of the overall system. The main aim is to show maximum power can be delivered, and fabricates a prototype if technology was available in Lebanon..
\end{abstract}

\section{INTRODUCTION}

Excessive time using the phone with excessive battery discharge is the main problem. So, capturing the RF Wi-Fi or mobile phone signals and transferring them to the maximum possible DC power with optimum system design, is a way to solve the problem and profit of power that is already available and lost.

Radio frequency (RF) energy harvesting method is becoming another method to drive power to the upcoming wireless networks. Nowadays, researchers from all over the world are concerned about energy and the environment. Energy harvesting is a technique to provide a real-time solution for alternative sources of energy that are ecofriendly, Non-conventional and low in cost. In near future, many wireless devices need to operate for longer durations away from conventional power sources. RF energy harvesting is one of the techniques that could enable us to harvest energy through converting radio frequency electromagnetic waves from ambient air to electrical energy.

Heinrich Hertz is the first researcher to introduce the first work of power transmission using the radio waves. His experiments are very closed to the projects and researches done in present years. He used at the transmitting and receiving of the system parts parabolic reflectors to propagate the electromagnetic waves in free space. The first power transmission research [1] was introduced in the form of rectenna (rectifying antenna). It worked between $2-3 \mathrm{GHz}$ band, and efficiencies $50 \%$ at output $4 \mathrm{~W}$ DC and $40 \%$ at output 7W DC respectively.
The modern history of this power transmission is based on developing the technology, methods and ways of microwave power transmission. main parts:

RF energy harvester system [2] will consist of 4

- Antenna [3]: Capture all radio frequency signals.

- Impedance Matching Circuit: Matching circuit used for antenna and usually it is LC circuit, and it's essential for transferring maximum RF power to the load.

- Rectifier [4]: Extract the power from the RF source, and convert it to DC voltage efficiently.

- Regulator [5]: Regulates voltage with low input value and increase the voltage.

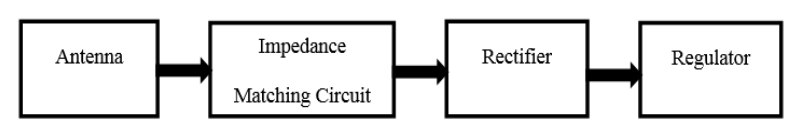

Figure 1: Block Diagram of RF Energy Harvesting System

Each part will be discussed and clarified. Also, the work will include a simulation for each part. For example, concerning the antenna part, simulations for antenna geometric shape, return loss, and radiation pattern using programs like HFSS are done. Moreover, the rectifier circuit and voltage converter will be drawn and simulated by programs.

The main goal is to show how much available power, the system can harvest from different sources like WiFi devices or GSM telephone band, etc.

\section{LITERATURE REVIEW}

After [1], many researches have been conducted [68] that lead to some advance in the domain, whether implemented or not, their results were a good start to trap energy released from mobile networks. Many operating frequencies for the antenna has been tested here. First the 2.45 $\mathrm{GHz}$ band has been implemented, since it's an available source form Wi-Fi networks. Second, it's the industrial, scientific and medical (ISM) band 5.8 GHz, due to the smaller shape and area of antenna compared to the $2.45 \mathrm{GHz}$, but at both frequencies similar advantages were monitored due to the low atmosphere loss, and components availability with low costs, and high efficiency conversion. Also, mobile telephone bands are good RF energy sources for harvesting energy, although they need a larger antenna size compared to the above-mentioned antenna sources. 


\section{IMPLEMENTATION/SIMULATION AND TESTING}

The design consists as previously mentioned of four main parts. first the antenna, where it is proposed and simulated. Second the matching circuit, between the antenna and rectifier to maintain maximum power harvested from the RF signals. After that, a rectifier to perform RF-DC conversion is simulated. At last, voltage doubling is proposed to multiply the harvested voltage to match output needs.

\section{Antenna design}

In this section, 3 microstrip patch antenna design are introduced. The three design have different substrate, shape, and resonant frequencies.

\section{Antenna - 1}

First design is showed below in Figure 2; the system is designed on HFSS program. The figure describes a circular patch antenna that was designed with modification to its shape on ADS. The patch is set at center frequency of 1.8 GHZ, with the use of RT-duroid 5880 substrate, that has a relative permittivity of 2.2 .

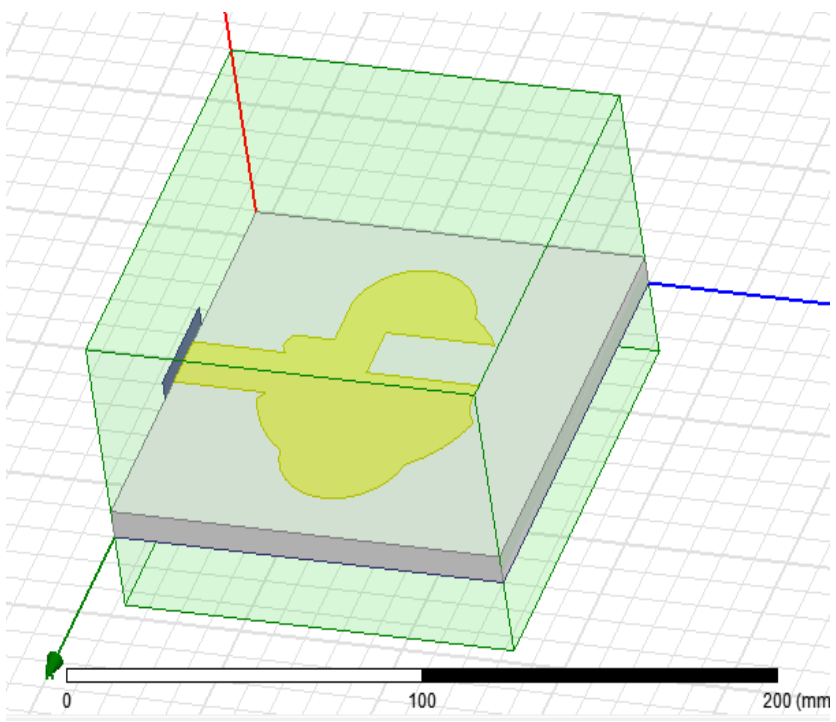

Figure 2: Modified Circular Patch Antenna

The patch and ground plane are set to perfect E metal conductivity, the small rectangle highlighted is set as wave port and the box as radiation pattern. The result is shown below.

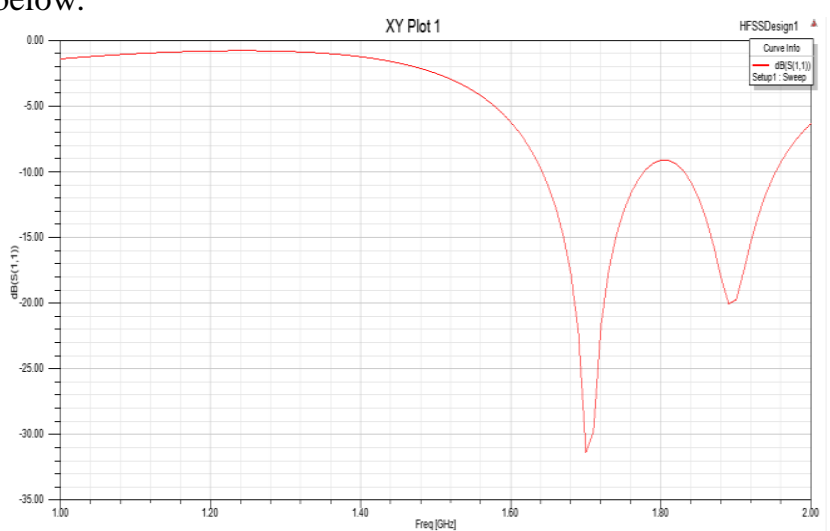

Figure 3: Return Loss Versus Frequency
Figure 3 shows, the return loss versus the frequency at the solution frequency $1.8 \mathrm{GHZ}$. At frequency $1.8 \mathrm{GHz}$ which is the desired value frequency to reached (one of the 3 basic frequencies), the antenna has the return loss around $-31 \mathrm{db}$.

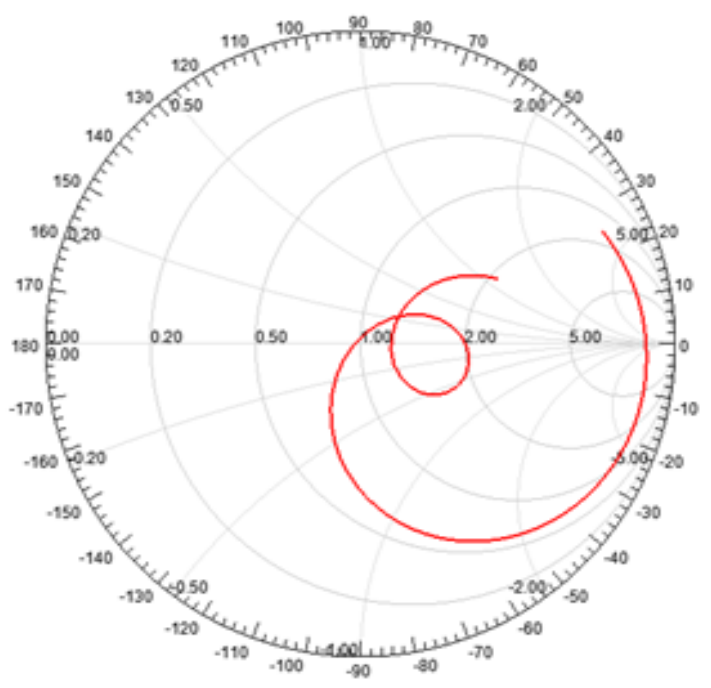

Figure 4: Smith Chart of 1.8 GHZ Antenna.

This smith chart shown in Figure 4 is a tool for visualizing the impedance of a transmission line and antenna system as a function of frequency in $1.8 \mathrm{GHZ}$ solution frequency antenna. Normally the input impedance is $50 \mathrm{ohms}$, if the value is different, the mismatch can be observed by the smith chart tool.

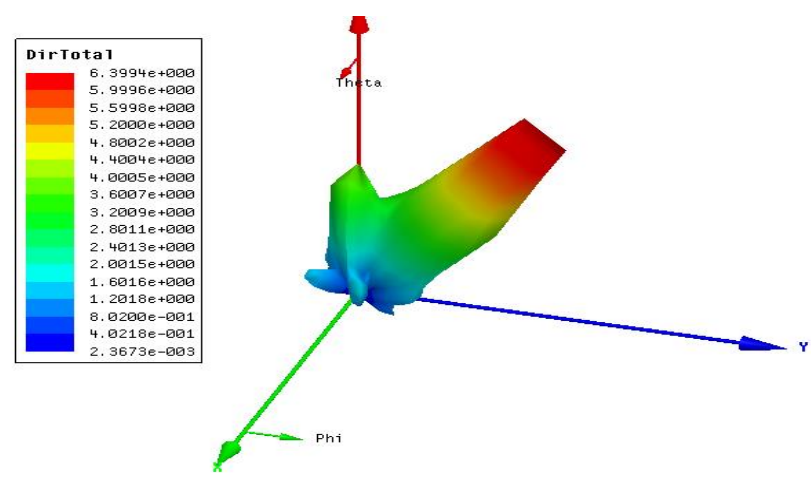

Figure 5: Directivity of Modified Circular Patch Antenna

Figure 5 shows the directivity of the simulated antenna.

Directivity is the ability of an antenna to focus energy in a particular direction when transmitting, or to receive energy better from a particular direction when receiving. In a static situation, it is possible to use the antenna directivity to concentrate the radiation beam in the wanted direction. In the figure, the red area shows high directivity. However, in a dynamic system here the transceiver is not fixed, the antenna should radiate equally in all directions, and this is known as an Omni-directional antenna. Gain representation using HFSS is shown in figure 6 of the modified circular antenna. 

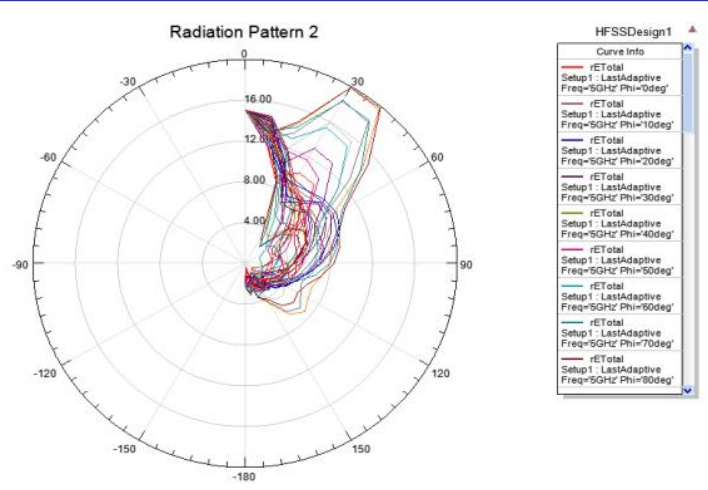

Figure 6: Radiation Pattern of 1.8 GHZ Antenna.

The radiation or antenna pattern describes the relative strength of the radiated field in various directions from the antenna, at a constant distance. The radiation pattern is a reception pattern as well, since it also describes the receiving properties of the antenna.

\section{Antenna - 2}

The second antenna is rectangular patch antenna also designed on HFSS. In this system, same explanation of the results is like the above but with different values of frequencies gains directivity and return losses.

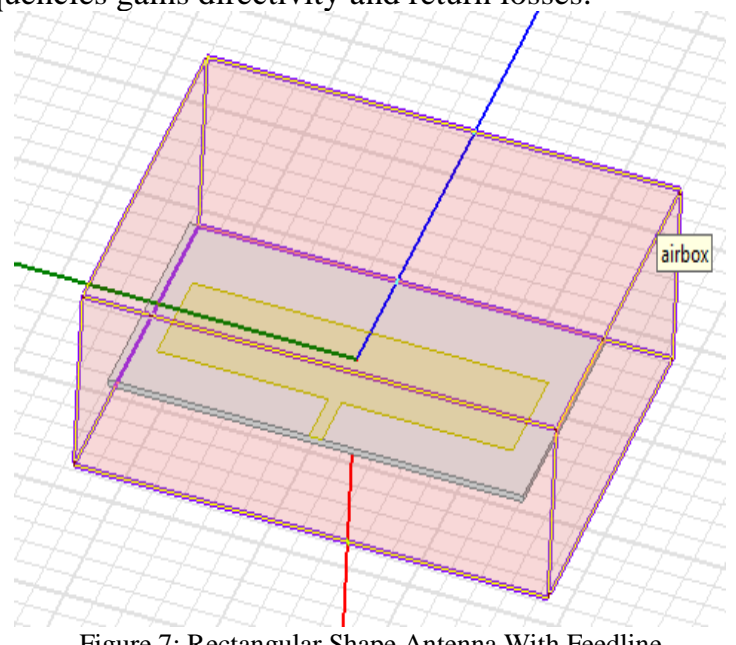

Figure 7: Rectangular Shape Antenna With Feedline

Figure 7 shows, a rectangular patch that was designed at a solution frequency of $2.45 \mathrm{GHZ}$ using " Arlon AD255A (tm) " substrate with Relative Permittivity reach 2.55. The rectangular patch was united with the feedline, and assigned with the rectangular plane as Perfect E metal, and the box as radiation pattern.

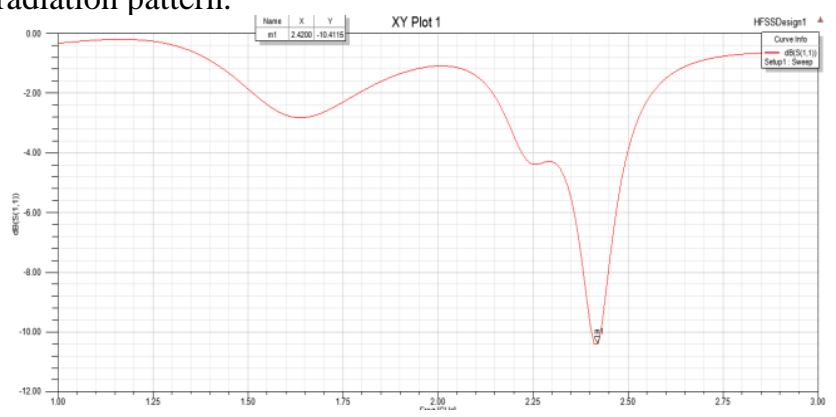

Figure 8: Return Loss Versus Frequency For Rectangular Antenna
Figure 8 shows, the return loss versus the frequency. At the frequency $2.4 \mathrm{GHZ}$ the power was $-11 \mathrm{db}$. As the width and length of the rectangular patch are modified, this implies with more enhanced results. The optimum solution with length of patch $=36.6 \mathrm{~mm}$ and width of patch $=120 \mathrm{~mm}$

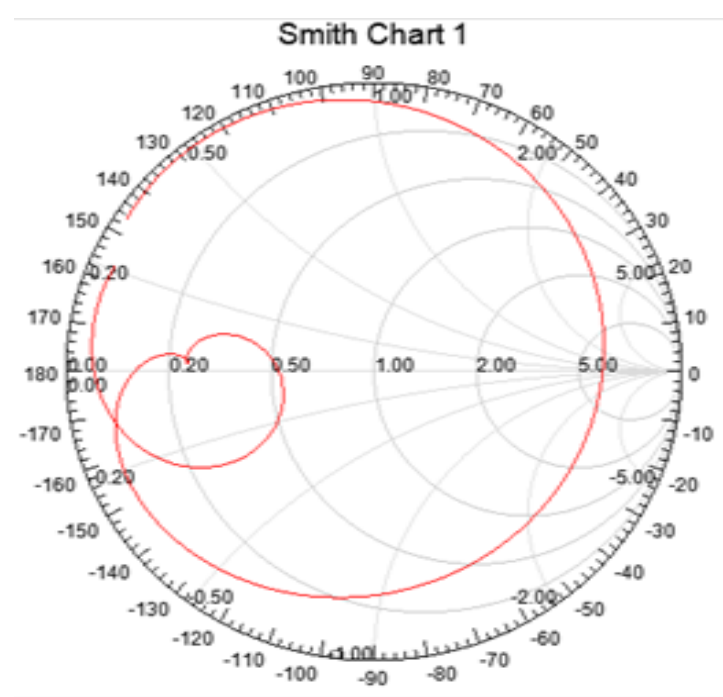

Figure 9: Smith Chart of 2.45 GHZ Rectangular Patch Antenna.

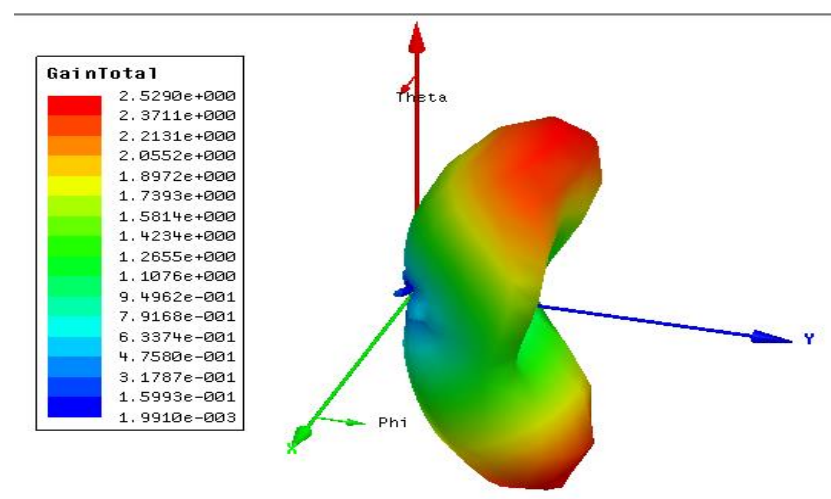

Figure 10: Total Gain of 2.4 GHZ Rectangular Patch antenna

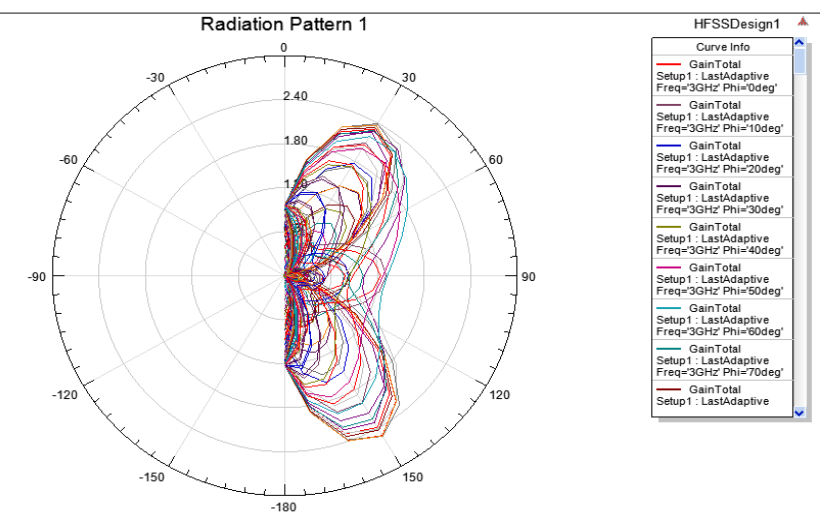

Figure 11:Radiation Pattern of 2.4 GHZ Antenna

Figure 10 shows the directivity and gain of the Rectangular patch antenna. While in figure 11 the radiation pattern is presented. 


\section{Simulation validation}

In order to verify the simulation done, another powerful electromagnetic simulator is used, which is momentum layout in ADS program.

Below down is the antenna design on ADS program. Its inserted as momentum layout.

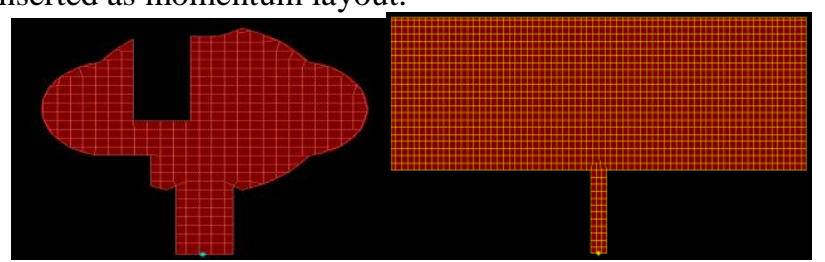

Figure 12: Momentum Layout of Circular Modified Patch Antenna and Rectangular Shape Antenna on ADS

In figure 12, the designed antenna drawn in ADS-momentum layout.

The results are shown below (figures 13 and 14).

In figure 4-16, antenna has its frequency at near $2.4 \mathrm{GHZ}$ solution. The results showed above are simulated as momentum microwave which is very accurate. If the results are compared to that of HFSS results, they are similar.

$$
\mathrm{S}(1,1) \quad \begin{aligned}
& \text { Calculated model data } \\
& \text { Adaptively fitted model data }
\end{aligned}
$$
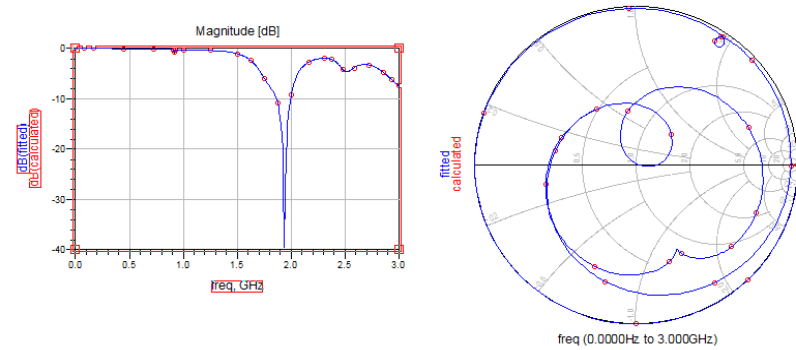

Figure 13: Result of Momentum Layout Analysis.

Similarly, in figure 14 the results of return loss versus frequency graph and the smith chart, as shown in the graph the momentum layout of circular modified patch antenna has a return loss around $40 \mathrm{~dB}$, and the smith chart corresponding to it.

In this layout analysis, the results obtained are similar to the results obtained from HFSS analysis.

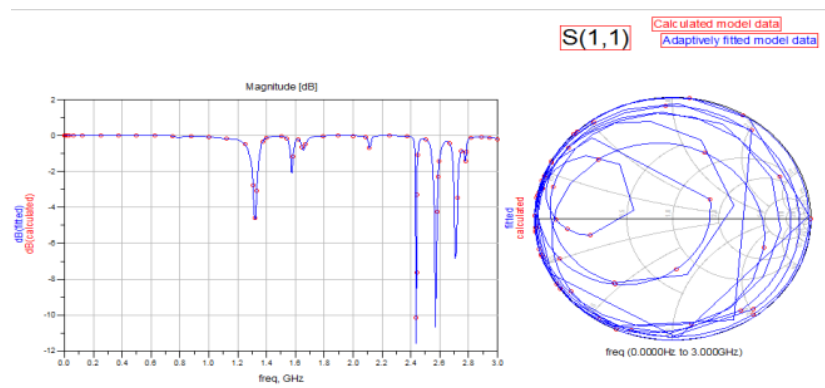

Figure 14: Rectangular and Impedance Matching Results
If the 2 antennas are compared, the rectangular antenna patch has many resonances. The modified circular patch antenna has approximately no resonance other than at its cnter frequency at $1.8 \mathrm{GHZ}$. It will be adopted for the next stage as the main input for the voltage doubler rectifier and impedance matching simulations.

Rectifier and voltage doubler.

The voltage doubler simulation consists of the rectifier combined with the voltage doubler and impedance matching network LC.

The circuits are designed on the ADS program. Schottky Diodes are to be used in the design. As known these days, Schottky diodes forward voltage (dropout) varies from 0.15 to 0.45 volt. In the model above, the assumed voltage drop is 0.15 volt, and very low current.

The designed system below (figure 15), are to assure that the voltage doublers and rectifications we are designing are possible. The circuit includes, sine wave source with amplitude of $200 \mathrm{mV}$ (the harvested assumption voltage according to the papers simulations) with frequency at 1 GHZ. The matching circuit LC with its values below (L6 and $\mathrm{C} 12$ ), and the doubler and rectifier circuit doubler stage combined together. The output voltage reaches the input voltage which is $200 \mathrm{mv}$. The result voltage is $200 \mathrm{mv}$.

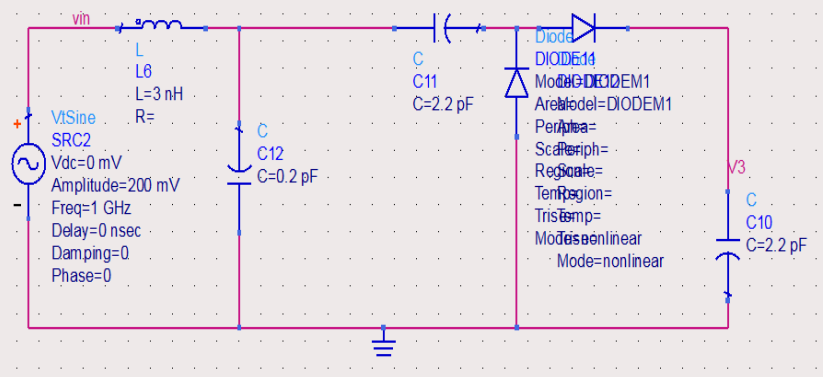

Figure 15: Single Stage Voltage Doubler

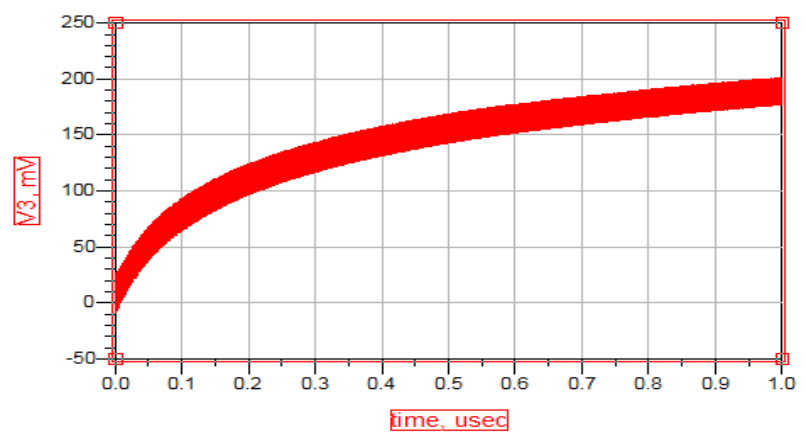

Figure 16: Output Voltage of Single Stage Voltage Doubler.

Four Stage Voltage Doubler

It is 4 single stage voltage doubler circuits combined together (figure 17). 


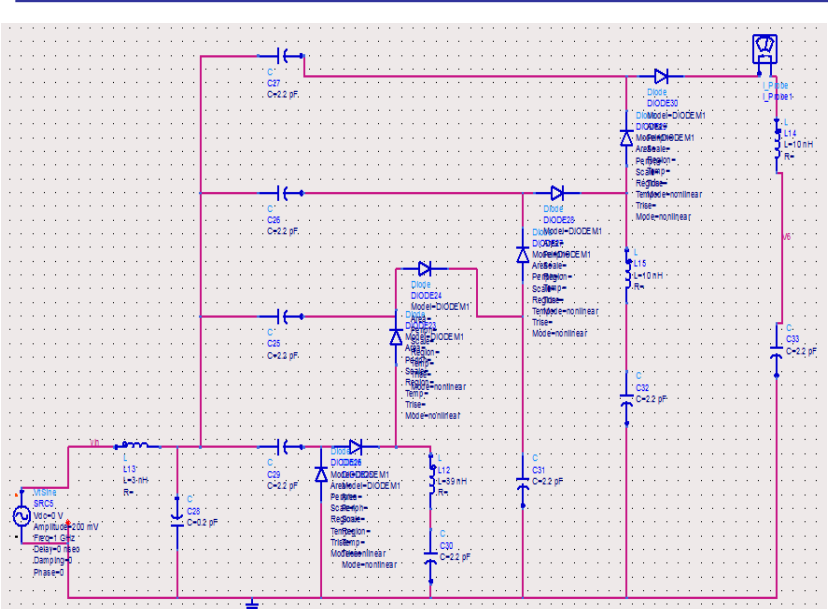

Figure 17: Fourth Stage Voltage Doubler.

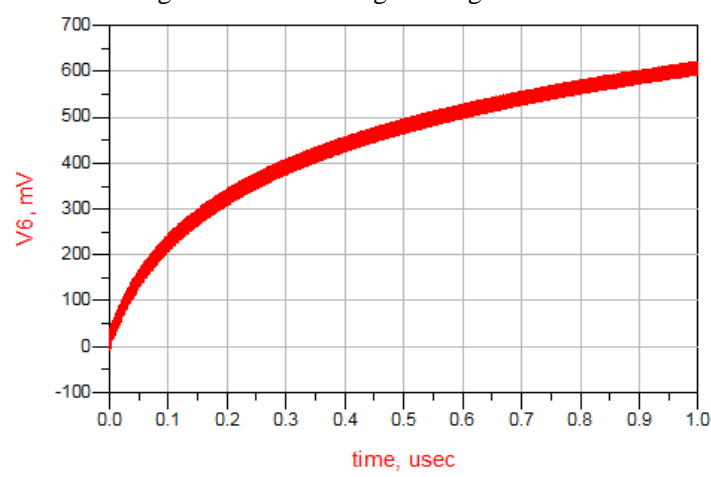

Figure 18: Curve of Fourth Stage Voltage Doubler.

The result of figure 18 shows that the obtained output voltage is $600 \mathrm{mV}$, while it should be $800 \mathrm{mV}$.

The 4 different voltage doublers (one to four stages) are presented in the plot below,

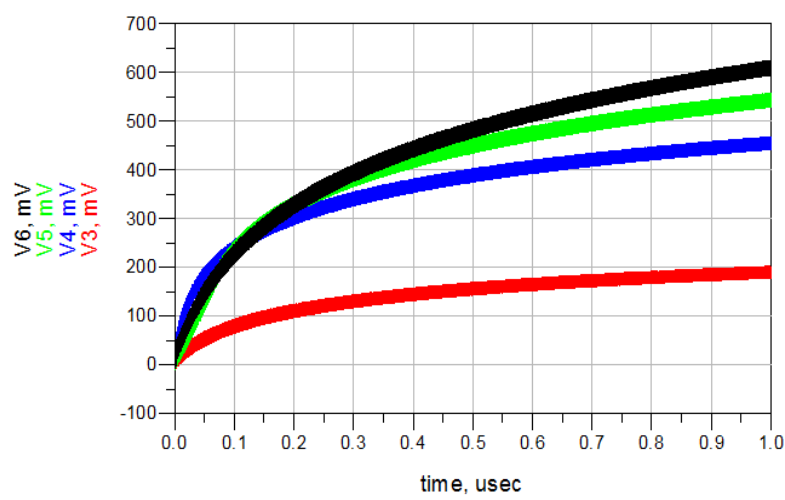

Figure 19: Output Voltages of the Voltage Doubler Circuits

The importance of this curve, is to show that, at some point the output voltage will not exceed the $600 \mathrm{mV}$ no matter how many stages are done after this. The idea is that the last stage shows that this is the maximum voltage that can be obtained so if we start to doubles the circuit to more stages the added obtained voltage will be reduced and reduced. For example, in stage 3 is $550 \mathrm{mv}$ in stage 4 its $600 \mathrm{mv}$.

Where between higher stages there is $100 \mathrm{mv}$ difference, and between lower stages there is $150 \mathrm{mv}$ difference.
Impedance matching design

In figure 20, the smith chart tool build in ads program is used to implement the impedance matching between source and the load. The source impedance is fixed at $50 \mathrm{ohms}$, where the load impedance by calculations is $=4.96872-88.734 * \mathrm{j}$, and the frequency is set to $1.8 \mathrm{GHZ}$. After that, we placed our impedance matching circuit which is LC circuit, and select auto-match between the 2 elements.

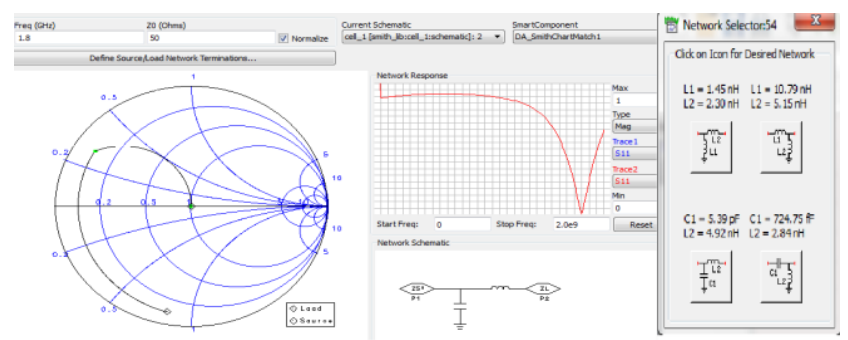

Figure 20: Smith Chart Impedance Matching for Single Stage Voltage Doubler

The results are shown in the figure above where we have four choices, the corresponding one that need to be used is the 3 result.

\section{SYSTEM DESIGN}

The Overall System is shown in the figure 21 below:

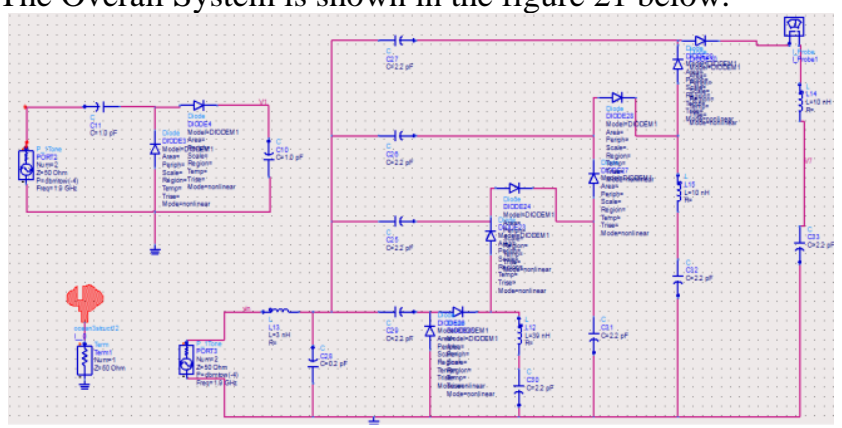

Figure 21: The Modified Patch Antenna and the Single and Multi Stage Voltage Doubler.

This figure shows the three circuits:

- The modified patch antenna connected to RF port with impedance $=50 \mathrm{ohm}$. The result of the return loss is shown below (figure 22)

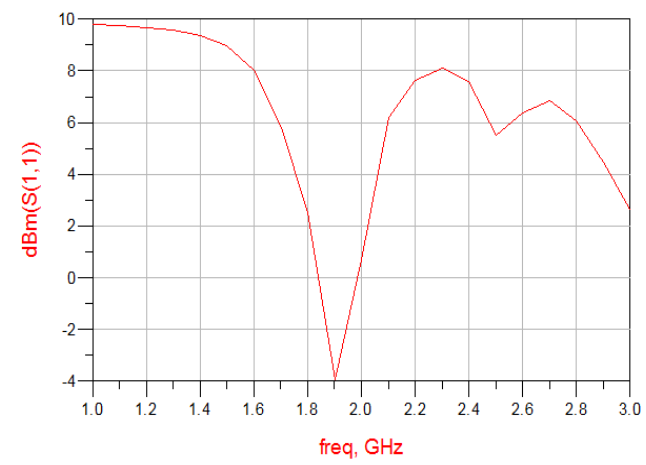

Figure 22: dBm S11 Return Loss.

As shown in figure 22 that at $1.9 \mathrm{GHz}$ solution frequency the antenna return loss is $-4 \mathrm{dBm}$. So, in the RF sources in voltage doublers circuits must be $-4 \mathrm{dBm}$ at $1.9 \mathrm{GHZ}$ 
From figure 23, the harvested voltage at the multistage doubler was 3.5 volt and the current is $1.5 \mathrm{~mA}$.

The maximum power obtained is $=1.5 \mathrm{~mA}^{*} 3 \mathrm{v}=4.5 \mathrm{~mW}$ This power is achieved if the power is at $-4 \mathrm{dbm}$, and nearby the source and no resonance and noises.

This result is simulation based results. In other words, the $4 \mathrm{dBm}$ antenna return loss is the perfect ideal case where the RF system is near the source with no noise and losses besides it, and at the best rectification efficiencies and no errors. In real measurement, this voltage may be dropped to 60 or 70 $\%$, but still the harvested voltage done as other experiments.
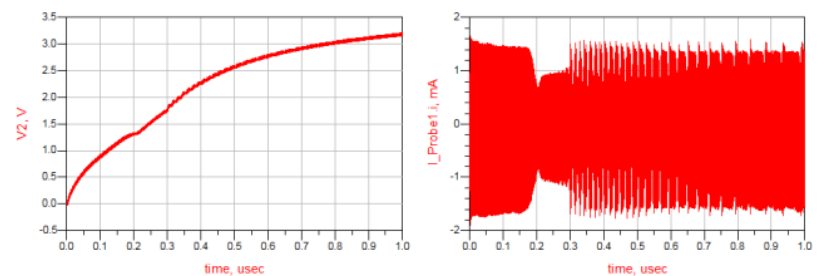

Figure 23: Results of RF Harvested Voltage and Current.

The current as shown is too small, but the application that needed to be built on RF harvested system should also need small current.

\section{CONCLUSION}

In RF harvesting, antenna need to be studied and designed well, because the antenna is source of the technique, so as much the design is accurate more power can be harvested. After that, the matching between source and load must be calculated especially for the load. and designed perfectly so to have realistic and maximum power transfer. Then, rectification and regulation must depend on high frequency diodes like Schottky due to their high efficiencies and low losses. Multi stages regulators are preferred due to their higher voltage and current values. And at last, simulations only are not enough; The disadvantages of this system design, it lacks the real measurements due to the absence of the prototype, and the needed technology. The simulations results are perfect, but with real life measurements more accurate results are achieved. In addition to this, multiband antenna which can harvest from one to three sources could be very efficient to implement.

\section{REFERENCES}

[1]. O. A. C. Escala, "Study of the efficiency of rectifying antenna systems," Universidad Politécnica de Catalunya, Barcelona, October 2010.

[2]. D. P. Taylor, "Radio Frequency Energy Harvesting Project," 2012.

[3]. D. O. a. G. Moernaut, "The Basics of Patch Antennas," RF Globalnet, p. 20, 2009.

[4]. S. Hemour, "Radio-Frequency Rectifier for Electromagnetic Energy Harvesting: Development Path and Future Outlook," IEEE, vol. 102. No.11, p. 25, 2014.

[5]. F. Yuan, "Chapter 2," in CMOS Circuits for Passive Wireless Microsystems, Springer Science+Business Media, 2011, p. 42.

[6]. N. Narampanawe, "Ambient Radio Frequency Energy Harvesting," Research Gate, p. 6, 2016.

[7]. B. Pham, "Triple Bands Antenna and High Efficiency Rectifier Design for RFEnergy Harvesting at 900, 1900 and $2400 \mathrm{MHz}$," Research Gate, p. 4, 2016.

[8]. P. D. M. L. Manuel Piñuela, "Ambient RF Energy Harvesting in Urban and Semi-Urban Environments," IEEE, Vols. 61,N0.7, p. 12, 2013. 